\title{
What is chronic constipation? Definition and diagnosis
}

\author{
James R Gray MD FRCPC
}

JR Gray. What is chronic constipation? Definition and diagnosis. Can J Gastroenterol 2011;25(Suppl B):7B-10B.

Although chronic constipation is a common complaint, there are no ideal biological markers for its diagnosis. Diagnosis rests on individual patient perception or clinical criteria developed by expert consensus. Investigation options are multiple but often not necessary. When selected, investigations are directed at confirming the absence of a primary underlying disease, differentiating or defining an alteration in colonic motility or identifying an anorectal evacuation disorder.

Key Words: Constipation; Definition; Diagnosis; Investigations

Onstipation is a very common concern, with complaints from those with the condition directed at family physicians and specialists, as well as pharmacists, nurses and family members, leading to a substantial cost to the health care system $(1,2)$. Constipation may be acute or chronic, with the latter usually being defined as a duration of greater than three months.

\section{DEFINITION}

There are no ideal disease markers for constipation; therefore, history becomes the major determinant of the diagnosis. Many definitions have been used, from a self-reported sense of constipation to the formal criteria used in clinical trials. This variety of definitions results in a wide range of reported prevalence (3-6). A practical definition, used by many patients, would simply be a reduced frequency or ease of stool passage from what is deemed the normal or expected pattern for that individual. Thus, patients will complain of reduced stool frequency or straining based, most often, on their own perception of what is normal or usual for them. It should be noted that self-reported frequency of bowel movements (BMs) is often underestimated unless stool diaries are used. In fact, in one study, $62 \%$ of patients reported themselves to be constipated while having one or more BMs per day (7). This may reflect behavioural issues such as stool withholding and reluctance to use unfamiliar toilet facilities.

Some definitions of chronic constipation include etiological considerations such as constipation secondary to medications, and/or neurological or systemic illnesses (Table 1). Most commonly, chronic constipation is deemed primary or idiopathic.

Over time, some previously considered causes of chronic constipation, such as dolichocolon (an elongated - but not dilated - colon) or chronic laxative use, have been discounted $(8,9)$.

The Rome III expert consensus definition of chronic constipation includes the presence of two or more of six symptoms (Table 2) (10). This definition should be distinguished from that of irritable bowel syndrome (IBS), in which pain becomes a requisite component symptom (Table 2).

The American College of Gastroenterology Chronic Constipation Task Force defined chronic constipation as "unsatisfactory defecation characterized by infrequent stool, difficult stool passage or both at least for previous 3 months". Difficult stool passage includes straining, a sense of difficulty passing stool, incomplete evacuation, hard/lumpy

\section{Qu'est-ce que la constipation chronique? Définition et diagnostic}

Même si la constipation chronique est un problème courant, il n'existe pas de marqueurs biologiques idéaux pour poser ce diagnostic. En fait, le diagnostic repose sur la perception du patient ou les critères cliniques élaborés par des consensus d'experts. Les examens possibles sont multiples, mais souvent inutiles. Lorsqu'ils sont choisis, les examens visent à confirmer l'absence d'une maladie sous-jacente primaire, à différencier ou à définir une modification de la motilité du côlon ou à déceler un trouble d'évacuation anorectale.

\section{TABLE 1}

Selected causes of secondary constipation

\begin{tabular}{l} 
Drugs \\
Narcotics \\
Anticholinergics \\
Antidepressants (especially tricyclic antidepressants) \\
Antipsychotics \\
Anti-Parkinson's medications \\
Calcium-channel blockers \\
Aluminum (antacids) \\
Metabolic conditions \\
Hypercalcemia \\
Severe hypothyroidism \\
Diabetes mellitus (with autonomic neuropathy) \\
Hypokalemia \\
Central nervous system diseases \\
Parkinson's disease \\
Multiple sclerosis \\
Spinal cord injury \\
Depression \\
Enteric myopathies or neuropathies \\
Scleroderma \\
Chronic pseudo-obstruction \\
Amyloidosis \\
Organic colorectal diseases \\
Rectocele \\
Cancer \\
Strictures (postinflammatory, postischemic, postdiverticulitis) \\
\hline
\end{tabular}

Adapted from reference 9

stool, prolonged time to stool or need for manual manoeuvres to pass stool" (Table 3) (11).

A Canadian consensus group defined chronic constipation as being "symptom based including fewer than 3 stools per week, stool form that is mostly hard or lumpy and difficult stool passage (need to strain or incomplete evacuation) for more than 6 months" (9).

Division of Gastroenterology, University of British Columbia, Vancouver, British Columbia

Correspondence and reprints: Dr James R Gray, Division of Gastroenterology, University of British Columbia, 5153-2775 Laurel Street, Vancouver,

British Columbia V5Z 1M9. Telephone 604-875-5618, fax 604-875 5373, e-mail allgray@telus.net

Received for publication April 8, 2011. Accepted June 10, 2011 


\section{TABLE 2}

\section{Rome III diagnostic criteria*}

Irritable bowel syndrome (10)

Recurrent abdominal pain or discomfort ${ }^{\dagger}$ at least 3 days per month in the previous 3 months associated with 2 or more of the following:

1. Improvement with defecation

2. Onset associated with a change in frequency of stool

3. Onset associated with a change in form (appearance) of stool

Functional constipation (10)

1. Must include 2 or more of the following:

a. Straining during at least $25 \%$ of defecations

b. Lumpy or hard stools in at least $25 \%$ of defecations

c. Sensation of incomplete evacuation for at least $25 \%$ of defecations

d. Sensation of anorectal obstruction/blockage for at least $25 \%$ of defecations

e. Manual manoeuvres to facilitate at least $25 \%$ of defecations (eg, digital evacuation, support of the pelvic floor)

f. Fewer than 3 defecations per week

2. Loose stools are rarely present without the use of laxatives

3. There are insufficient criteria for irritable bowel syndrome

Functional defecation disorder (27)

1. The patient must satisfy diagnostic criteria for functional constipation (above).

2. During repeated attempts to defecate, the patient must have at least 2 of the following:

a. Evidence of impaired evacuation, based on balloon expulsion test or imaging;

b. Inappropriate contraction of the pelvic floor muscles (ie, anal sphincter or puborectalis) or less than $20 \%$ relaxation of basal resting sphincter pressure by manometry, imaging or electromyography

c. Inadequate propulsive forces assessed by manometry or imaging.

${ }^{*}$ Criteria fulfilled for the previous three months with symptom onset at least six months before diagnosis; ${ }^{\dagger}$ Uncomfortable sensation not described as pain Numbers in parentheses indicate reference

\section{EVALUATION}

A careful history and physical examination are most important in the evaluation of a patient complaining of constipation. The history should include an understanding of the patient's perception of their current bowel habit compared with the past, perhaps aided by a twoweek stool diary that would include stool frequency, character or shape, and passage of stool. Use of the Bristol Stool Scale may serve as a template to describe stool form (Table 3) (12). One should gain an understanding of what measures, including type, dose and duration of laxatives, were previously tried. It is useful to understand the concerns and expectations the patient has for their bowels, and what goals they have for their bowel pattern. Many are simply seeking reassurance.

A search for 'alarm' features such as unintentional weight loss, rectal bleeding or new onset of symptoms, especially after 50 years of age, is required. The history should include a drug history, both prescribed and over the counter, and a review for systemic or neurological disorders that may be associated with constipation (Table 1). History should also help to differentiate IBS from chronic constipation, with the former typically including constipation alternating with diarrhea and, most distinctively, including pain associated with bowel movements (Table 2).

Physical examination should include palpation of the abdomen in search for palpable stool indicating fecal loading. A careful rectal examination is critical. This may identify evidence of anal diseases such as strictures, fissures, inflammation, rectocele or masses. Neurological disorders may manifest as a gaping or patulous anus or reduced perianal sensation. Failure of perineal descent when the patient bears down suggests pelvic floor dysfunction $(13,14)$.
TABLE 3

\section{Bristol Stool Scale}

\begin{tabular}{ll}
\hline Type & Description \\
\hline 1 & Separate hard lumps, similar to nuts (difficult to pass) \\
2 & Sausage shaped but lumpy \\
3 & Like a sausage but with cracks on its surface \\
4 & Like a sausage or snake, smooth and soft \\
5 & Soft blobs with clear cut edges (passed easily) \\
6 & Fluffy pieces with ragged edges, a mushy stool \\
7 & Watery, no solid pieces, entirely liquid
\end{tabular}

Data adapted from reference 28

From the history and physical examination one can usually identify or suspect those with new-onset or secondary constipation due to medications, and/or neurological or endocrinological diseases, IBS or those with obstructive pathology such as cancer. The remainder would be diagnosed with primary or idiopathic constipation.

\section{INVESTIGATIONS}

The role of the colon is to absorb water and to deliver stool to the rectum from where it can be evacuated in a comfortable fashion. Constipation can arise from disorders of transit along the colon or from disorders of evacuation from the rectum through the anus. Tests to evaluate each of these physiological components are available, although none with an ideal sensitivity or specificity (15).

Many tests for the evaluation of primary constipation are performed in clinical practice; however, these are often not revealing and, thus, not very useful in recognizing that physiological abnormalities are seldom appreciable in chronic primary constipation. Therefore, a therapeutic trial of fibre with or without simple laxatives is often appropriate, and will suffice after the initial and, presumably, normal history and physical examination. If successful, it may offer reassurance to the patient and physician, and preclude the need for any additional investigations (16).

The American College of Gastroenterology Chronic Constipation Task Force "did not endorse the routine use of a battery of diagnostic tests ... and acknowledged that there is inadequate evidence to make a strong recommendation about the appropriate diagnostic approach to patients with chronic constipation symptoms" (11).

The Canadian Association of Gastroenterology consensus on chronic constipation (9) agreed that "testing to rule out organic disease should be left to the judgement of the treating physician based on symptom severity, the impact on the patient's quality of life, and the presence of risk factors or alarm features obtained from the history and physical examination". However, this latter group believed that there was value in classifying chronic constipation into its motility pattern, implying the need for a colonic transit study. The three colon transit study outcomes include slow transit, normal transit or obstructed defecation, with a distribution of $13 \%, 59 \%$ and $25 \%$, respectively, with an overlap among the remainder (9).

It should be noted that none of the expert consensus definitions of chronic constipation require investigations that include colon transit studies. However, the Rome III criteria for dyssynergic defecation requires two objective findings combined with the criteria for functional constipation (Table 2) (17).

Many have used colon transit time as an initial differentiating point, which of course requires a measure of colon transit usually by using radiopaque markers and a subsequent timed abdominal radiograph (see below). From this, individuals can be separated into those with normal colon transit, slow transit or dyssynergic defecation, ie, disorders of stool movement through the colon or disordered evacuation from the anorectum. Unfortunately, there is some overlap in the distribution of these subtypes. For example, many patients with dyssynergic defecation will also demonstrate slow transit (17).

When colonic transit is measured, there is wide variation in subtype distribution patterns, perhaps reflecting sample selection 
(including age and sex) as well as test characteristics among various centres (18). This variation and the degree of overlap has raised questions about the place for and value of transit studies in the absence of large prospective studies $(17,18)$.

Disorders of evacuation of stool from the rectum appear under complex terminology that includes dyssynergic defecation, pelvic floor dysfunction, anismus, dyschezia, outlet delay or outlet obstruction, and embrace a range of diseases (19). The Rome III diagnostic criteria for dyssynergic defecation are listed in Table 2.

The clinical hallmarks of dyssynergic defecation are prolonged and excessive straining, often leading to vaginal or perianal pressure being applied to facilitate evacuation.

Normal defecation requires coordination between the rectal sensing of accumulating stool with increasing rectal pressure, and subsequent pelvic floor and anal sphincter relaxation in conjunction with personal interpretation of social acceptability to attend the toilet or pass stool. The anorectal components can be measured using anorectal manometry (see below), but are limited by relatively poor standardization and the potential effects of extracolonic problems including sexual abuse and psychological distress along with patient comfort issues attendant in the performance of these tests $(19,20)$. One study $(21)$, in distinction to others, found that as many as $50 \%$ of patients with refractory constipation studied in a prospective fashion demonstrated evidence of obstructive defecation.

Thus, the value and choice of testing remains unclear in most patients with chronic idiopathic constipation. However, there are many test options, some of which are summarized in the following section.

\section{TESTS}

\section{Laboratory tests}

Serum thyroid function, glucose, electrolytes and calcium, complete blood count and urinalysis are often recommended, but there is no literature to support their usefulness in the workup of patients with chronic constipation (15).

\section{Endoscopy}

In the absence of alarm features, such as bleeding or new-onset constipation, there is no increased diagnostic yield with using either flexible sigmoidoscopy or colonoscopy beyond what would be expected in assymptomatic individuals undergoing colorectal cancer screening (22). However, one may consider and offer age- and interval-appropriate screening to patients with constipation as one would to asymptomatic individuals.

\section{Radiological tests}

Plain abdominal $\mathrm{x}$-ray: In spite of the common use of plain abdominal $\mathrm{x}$-rays to assess 'fecal loading', there is no literature supporting it. In fact, there is no standard measure of a 'normal' fecal load from which to compare.

Barium enema: There is no current literature to support the use of barium enemas in the workup or evaluation of patients with chronic constipation. Presently, these are rarely performed and have been replaced by endoscopy or computed tomography colonography, where there is a need to rule out obstructive pathology within the colon.

Endoscopic ultrasound: While useful in assessing the integrity of the anal sphincter and extrarectal or presacral space, it has not yet found a place in the evaluation of constipation.

Defecography: This test, not usually performed in clinical practice due to patient discomfort and the lack of standardized techniques or measurements, is designed to reveal anatomical variations in the anorectum that could contribute to constipation from outlet delay. Barium paste is placed in the rectum to mimic natural stool consistency and fluoroscopically observed to be passed by the patient on a specially designed and fluoroscopically accessible commode (23). Various measurements of the movement of the pelvic floor and rectum can be taken during this process. In particular, this test should demonstrate the normal 'opening' of the anorectal angle and proper pelvic floor descent during the act of defecation. Absence of these changes suggests dyssynergic defecation. Its only use is in defining the anatomical and functional anatomy of the anorectum after dyssynergic defecation is already identified, and should not be used as an initial or sole test in the evaluation of constipation $(12,15)$.

\section{Magnetic resonance imaging}

Similar to endoscopic ultrasound, magnetic resonance imaging is invaluable in the assessment of the perirectal tissue planes; however, to date, it is not useful or readily available in assessing functional constipation (23).

\section{Physiological testing}

These tests are the only investigations that actually attempt to measure the physiological movement of stool within the colon and rectum.

Colon transit study: While consistently consuming a high-fibre diet and avoiding laxatives, an individual swallows several radiopaque markers that are followed through the colon with plain $\mathrm{x}$-rays. A variety of techniques have been proposed since they were originally described in 1969 (24). The simplest, requiring the least radiation exposure, involves ingesting a commercially available capsule containing the 24 markers (this can be made by introducing $242 \mathrm{~mm}$ to $3 \mathrm{~mm}$ slices of a radiopaque nasogastric tube in gelatin capsules) and obtaining an abdominal $x$-ray after six days. With 'normal transit', less than five markers will remain in the colon; with 'slow transit', six or more markers will remain and be scattered throughout the colon. The third pattern, indicating dyssynergic defecation or outlet delay, will be revealed by finding six or more markers clustered in the rectosigmoid (17).

Although a simple and relatively inexpensive test that is widely performed, radiopaque marker studies have limitations (25). First, they actually measure total oral-anal transit, not just colon transit. Second, the definition of 'normal' is based on a limited number of studied healthy subjects, with a broad range of results that are not well standardized (25). A systematic review of the literature (15) showed poor reproducibility for these tests, especially in patients with slow transit or dyssynergic defecation compared with those with normal transit, which indicates low specificity.

\section{Anorectal motility:}

Manometry: This technique is also a direct measure of physiological function - but only within the rectum - and enables the assessment of rectal compliance, rectal sensation and the presence of anorectal reflexes. Thus, its goal is the confirmation and assessment of outlet delay or dyssynergic defecation. Its greatest value is in ruling out Hirschsprung's disease. It is most recently reviewed in detail in the American Gastroenterological Association medical position statement on anorectal testing techniques (12). A more recent systematic review (15) underscored the poor sensitivity for anorectal manometry and lack of clear standards and definition for dysynergic defecation.

Balloon expulsion: First described in 1985 (26), this timed test assesses the ability of the patient to expel a standard-size object usually a $50 \mathrm{~mL}$ fluid-filled balloon - from the rectum. The balloon can usually be passed within $3 \mathrm{~min}$; failure to do so suggests dyssynergic defecation. There are a variety of protocols for this procedure, but it is limited by patient embarrassment and its usual performance in the left lateral decubitus position, which is far removed from the usual sitting position for defecation. The lack of standardization remains a weakness of this test.

Scintigraphy: This test uses nuclear medicine and measurement of the transit of radiolabelled stool through the colon. It is rarely performed outside of a research setting and, therefore, is not a clinically practical or available test to most clinicians (25).

Electrophysiology: These tests, including pudendal nerve terminal motor latency measurements and electromyography of the external anal sphincter or puborectalis, currently belong in the arena of clinical research (16). 


\section{SUMMARY}

Chronic constipation is a common problem that may be acute or chronic. Many definitions have been used. It may be idiopathic or primary, or secondary to a variety of medical or metabolic disorders. History and physical examination are the most important initial approaches to the evaluation of those with constipation. In the absence of any alarm features or suggestions of an associated disorder, a therapeutic trial with bulking agents or simple laxatives is an appropriate first step. Diagnostic investigations can be performed in a selective fashion directed at either defining the nature of transit through the colon or evacuation through the anorectum or confirming the absence of a primary disease leading to the symptoms of constipation.

CONFLICTS OF INTEREST: The authors have no financial disclosures or conflicts of interest to declare.

\section{REFERENCES}

1. Everhart JE, Ruhl CE. Burden of digestive diseases in the United States Part 1: Overall and upper gastrointestinal diseases. Gastroenterology 2009;136:376-86.

2. Wald A, Scarpignato C, Kamm MA, et al. The burden of constipation on quality of life: Results of a multinational survey. Aliment Pharm Therap 2007;26:227-36.

3. Garrigues V, Galvez C, Ortiz V, et al. Prevalence of constipation: Agreement among several criteria and evaluation of the diagnostic accuracy of qualifying symptoms and self-reported definition in a population based survey in Spain. Am J Epidemiol 2004;159:520-6.

4. Ashraf W, Park F, Lof J, et al. An examination of the reliability of reported stool frequency in the diagnosis of idiopathic constipation. Am J Gastroenterol 1996;91:26-32.

5. Everhart JE, Go VL, Johannes RS, et al. A longitudinal survey of self-reported bowel habits in the United States. Dig Dis Sci 1989;34:1153-62.

6. Johanson JF, Kralstein J. Chronic constipation: A survey of the patient perspective. Aliment Pharm Therap 2007;25:599-608.

7. Sandler RS, Drossman DA. Bowel habits in young adults not seeking health care. Dig Dis Sci 1987;32:841-5.

8. Muller-Lissner SA, Kamm M, Scarpignato C, et al. Myths and misconceptions about chronic constipation. Am J Gastroenterol 2005; 100:232-42.

9. Pare P, Bridges R, Champion M, et al. Recommendations on chronic constipation (including constipation associated with irritable bowel syndrome) treatment. Can J Gastroenterol 2007;21(Suppl B):3B-22B.
10. Longstreth GF, Thompson WG, Chey WD, et al. Functional bowel disorders. Gastroenterology 2006;130:1480-91.

11. Brandt LJ, Schoenfeld P, Prather CM, et al. An evidence-based approach to the management of chronic constipation in North America. Am J Gastroenterol 2005;100(Supp 1):S5-22.

12. Barnett JL, Hasler WL, Camilleri M. American Gastroenterological Association medical position statement on anorectal testing techniques. Gastroenterology 1999;116:732-60.

13. Talley NJ. How to do and interpret a rectal examination in gastroenterology. Am J Gastroenterol 2008;103:820-2.

14. Lembo A, Camilleri M. Chronic constipation. N Engl J Med 2003;349:1360-8.

15. Rao SSC, Ozturk R, Laine L. Clinical utility of diagnostic tests for constipation in adults: A systematic review. Am J Gastroenterol 2005;100:1605-15

16. Locke GR III, Pemberton JH, Phillips SF. American Gastroenterological Association medical position statement: Guidelines on constipation. Gastroenterol 2000;119:1761-78.

17. Rao SSC. Constipation: Evaluation and treatment of colonic and anorectal motility disorders. Gastroenterol Clin North Am 2007;36:687-711.

18. Cook IJ, Talley NJ, Benninga MA, et al. Chronic constipation: Overview and challenges. Neurogastroenterol Motil 2009;21(Suppl 2):1-8.

19. Lunnis PJ, Gladman MA, Benning MA, et al. Pathophysiology of evacuation disorders. Neurogastroenterol Motil 2009;21(Suppl 1):31-40.

20. Rao SC, Tuteja AK, Vellema T, et al. Dyssynergic defecation: Demographics, symptoms, stool patterns and quality of life. J Clin Gastroenterol 2004;38:680-5.

21. Rao SSC, Patel RS. How useful are manometric tests of anorectal function in the management of defecation disorders. Am J Gastroenterol 1997;92:469-75.

22. Pepin C, Ladabaum U. The yield of lower endoscopy in patients with constipation: Survey of a university hospital, a public county hospital and a Veterans Administration medical center. Gastrointest Endosc 2002;56:325-32.

23. Savoye-Collet C, Korning E, Dacher JN. Radiologic evaluation of pelvic floor disorders. Gastroenterol Clin North Am 2008;37:553-67.

24. Hinton JM, Lennard-Jones JE, Young AC. A new method for studying gut transit times using radioopaque markers. Gut 1969;10:842-7.

25. Dinning PG, Smith TK, Scott SM. Pathophysiology of colonic causes of chronic constipation. Neurogastroenterol Motil 2009;21(Suppl 1):20-30.

26. Preston DM, Lennard-Jones JE. Anismus in chronic constipation. Dig Dis Sci 1985;30:413-8.

27. Bharucha AE, Wald A, Enck P, et al. Functional anorectal disorders. Gastroenterol 2006;130:1510-8.

28. O'Donnell L, Virjee J, Heaton K. Detection of pseudodiarrhea by simple clinical assessment of intestinal transit rate. Br Med J 1990;300:439-40. 


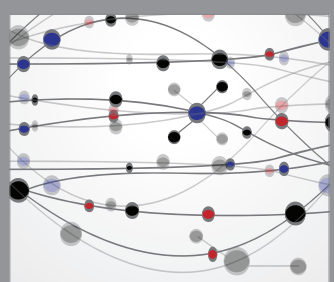

The Scientific World Journal
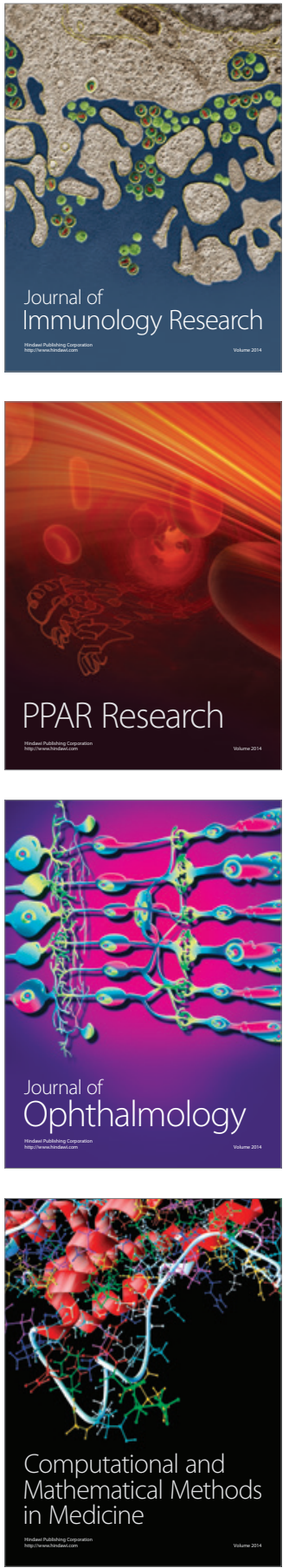

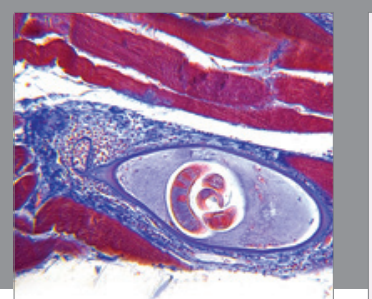

Gastroenterology Research and Practice

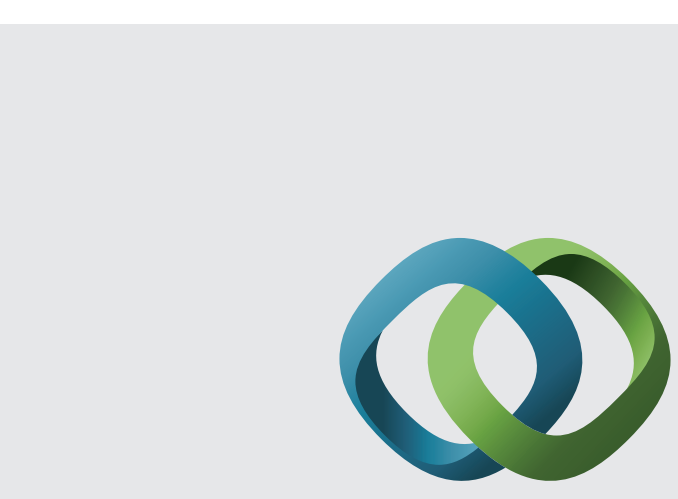

\section{Hindawi}

Submit your manuscripts at

http://www.hindawi.com
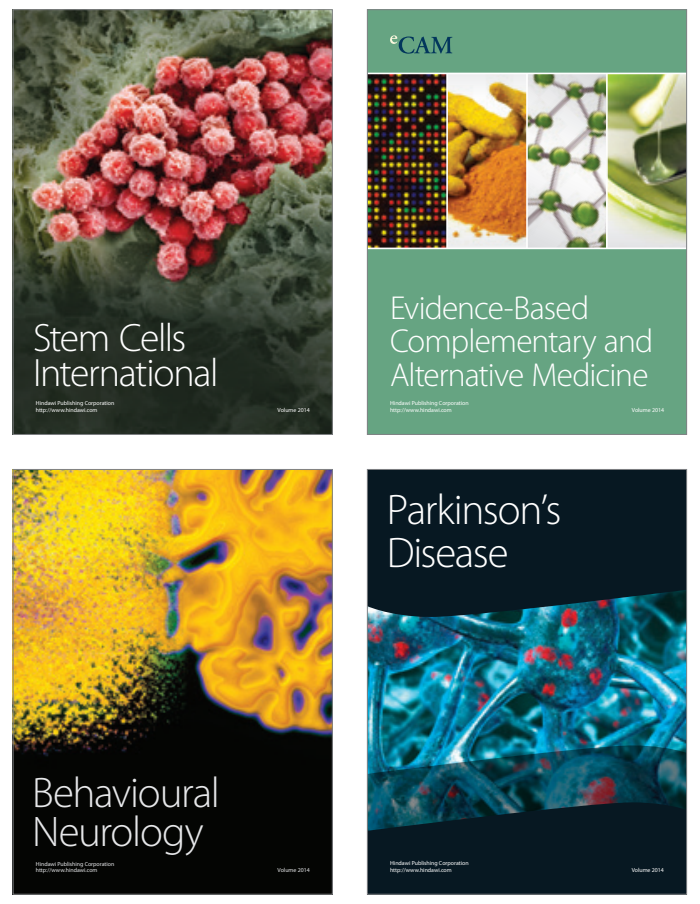
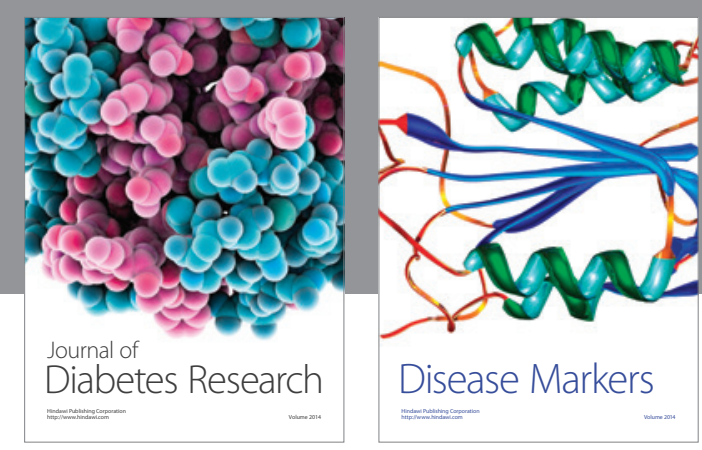

Disease Markers
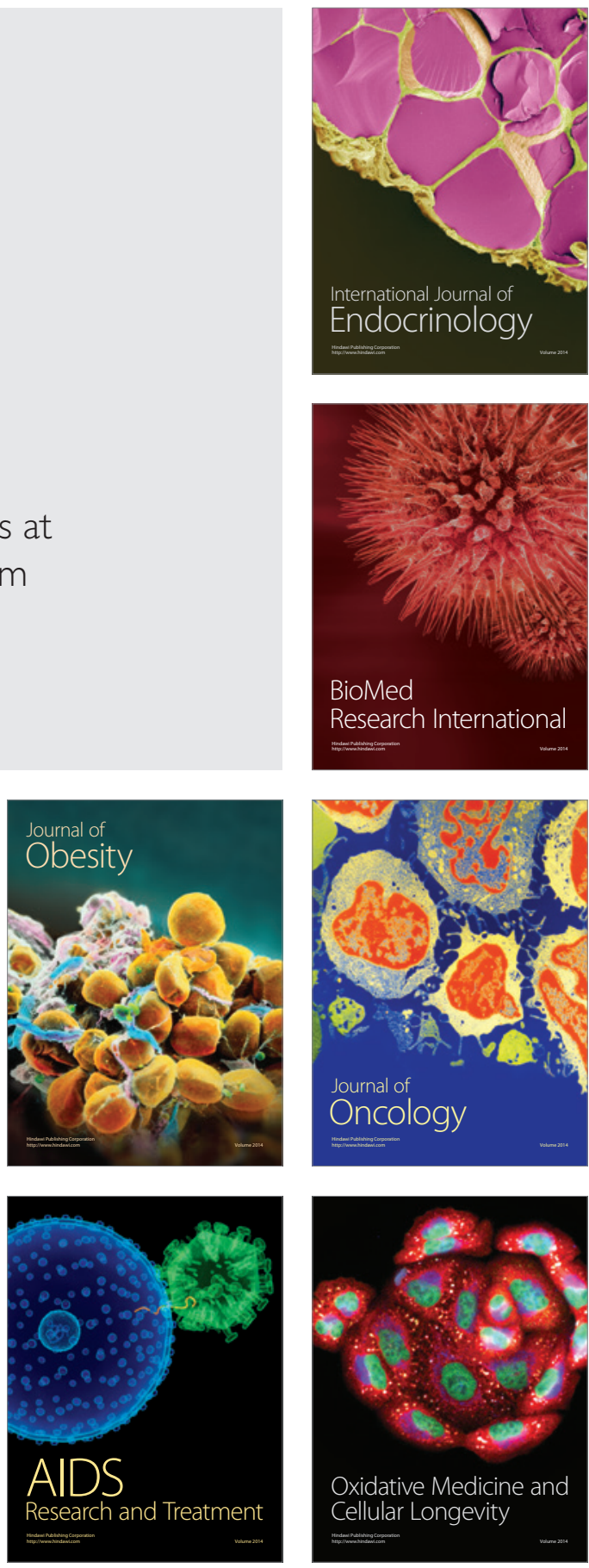